\title{
Guidelines for Performing Nuclear Magnetic Resonance Imaging Examinations in Patients with Cardiac Electronic Devices
}

\author{
Orientações para Realização de Exames de Ressonância Magnética Nuclear \\ em Pacientes com Dispositivos Eletrônicos Cardíacos
}

Antonio Vitor Moraes Junior ${ }^{1,2}$, Bruno Pereira Valdigem ${ }^{1,2^{*}}$, Cecilia Monteiro Boya Barcelos ${ }^{1,2}$, Celso Salgado de Melo ${ }^{1,2}$, Wilson Lopes Pereira ${ }^{1,2}$, Hilton Muniz Leão Filho3, Marco Antônio Rocha Mello $^{3}$, Cyro Antônio Fonseca Junior ${ }^{3}$, Fernando Eduardo Nunes Mariz ${ }^{3}$, Marcelo Rodrigues de Abreu $^{3}$, Patrícia Prando Cardia ${ }^{3}$, Paulo Roberto Vieira de Andrade ${ }^{3}$, Simone Kodlulovich Renha ${ }^{3}$, Alexander dal Forno ${ }^{4}$, Andre Luiz Buchele D' Avila4, Ricardo Alkmin Teixeira ${ }^{4}$, Veridiana Silva de Andrade ${ }^{4}$

\section{INTRODUCTION}

It is estimated that up to $75 \%$ of patients with cardiac implantable electronic devices (CIEDs) will have an indication for nuclear magnetic resonance imaging (MRI) throughout their lives. This population has been historically excluded from the list of patients considered eligible for this examination due to the characteristics of the devices.

A CIED consists of electrode leads and a generator. Each electrode lead is a multifilament metal spiral connector that connects the generator to the heart muscle. The generator, in turn, consists of battery, circuits and connector for the electrode leads. The function of the electrode leads is to conduct electrical impulses with minimum energy sufficient to initiate a cardiac electrical impulse ( $\mathrm{P}$ or QRS wave). Another essential function of the leads is to transmit electrical information acquired in the myocardium (intracavitary electrogram) to the generator, i.e., to feel the patient's native electrical rhythm, avoiding unnecessary stimulation.

The generator is usually located in the right or left infraclavicular space (or less frequently in the lateral region of the chest or abdomen). It has the function of interpreting stimuli coming from the electrode leads and generate electrical impulse by electric current between the myocardium and the generator (using the electrode leads as conductors). It contains the battery necessary for the impulse (with durability ranging from 7 to 15 years in general) and programmable circuits, that allow minimum frequency, increase in movement-dependent frequency, integration between the electrode leads located in several heart chambers, etc. In exceptional cases, such as in children, the generator can be positioned in the abdomen, and the electrode leads are usually transvenous and, eventually, epimyocardial. Systems with the generator directly implanted into the heart (leadless pacemaker) or with subcutaneous implantable cardioverter-defibrillator (ICD) are available for use in selected cases. The magnetic field generated by MRI can be interpreted by the CIED as abnormal cardiac electrical signal (QRS or P wave) and create interference that would cause one of the following behaviors:

1.Sociedade Brasileira de Cirurgia Cardiovascular - Departamento de Estimulação Cardíaca Artificial - São Paulo/SP - Brazil

2.Associação Brasileira de Arritmia, Eletrofisiologia e Estimulação Cardíaca Artificial - São Paulo/SP - Brazil

3.Colégio Brasileiro de Radiologia e Diagnóstico por Imagem - São Paulo/SP - Brazil

4.Sociedade Brasileira de Arritmias Cardíacas - São Paulo/SP - Brazil

*Correspondence author: valdigem@gmail.com

ORCID: Valdigem BP (iD https://orcid.org/0000-0003-0676-2033 
- Trigger artificial heart stimuli at high frequency;

- Inhibit stimuli of the heart;

- Damage the electrode leads, generator or heating of the system;

- Modification of forced stimulation parameters (reset);

- Trigger inappropriate shocks (in the case of internal cardioverter-defibrillator).

Despite the initial concern, the movement of the device and the torsion of the electrode leads were not evident due to the adherence of the tissues to the subcutaneous.

The heating of the system (causing damage to components and injury to the myocardium around the electrode lead) was proven for nonconditional electrodes. This may lead to an increase in the threshold necessary for myocardial stimulation and patient discomfort.

All CIEDs to be submitted to MRI shall be reprogrammed before and after the examination. Some newer devices are capable to detect the magnetic field of the MRI when activated for a predetermined period, which makes programming appropriate when the patient is inside the scanning room (zone 4). Most of them, however, should be reprogrammed to asynchronous or appropriate mode as late as possible and returned to the original setting after the end of the examination in the shortest period considered appropriate by the physician responsible for monitoring the patient with CIED. This orientation is based on the potential risk of asynchronous stimulation competing with the patient's own pace and being potentially arrhythmogenic in pacemakers. Patients with cardioverter-defibrillators should not leave a supervised environment without adequate protection from antitachycardia therapies.

\section{DEFINITION OF DEVICES}

- Pacemaker: device that has the function of stimulation and sensitivity. The pacemaker allows to guarantee the minimum stimulation frequency of the patient. Programming is described by letters (Table 1).

- Resynchronizers: also called multisite pacemaker, they are devices that allow simultaneous stimulation of the left ventricle (LV) by an electrode lead positioned in a epicardial tributary vein of the venous coronary sinus. They may or may not be associated with defibrillator function and, if so, it is called multisite defibrillator. Patients with cardiac resynchronizers have significant structural heart disease and compromised LV ejection fraction (usually less than 35\%). The LV electrode lead is positioned in a epicardial tributary vein of the venous coronary sinus. Eventually the LV electrode lead can be implanted by epimyocardial access.

- Cardioverter-defibrillators (ICDs): CIEDs with stimulation function identical to the pacemakers'. They also have a capacitor that allows the device to release shocks with high energy. Their function is to control ventricular tachycardia or ventricular fibrillation, and they are usually implanted in patients with different degrees of ventricular dysfunction or with higher risk of cardiorespiratory arrest.

- Event monitors: Subcutaneous devices from 3 to $6 \mathrm{~cm}$ positioned in the anterior thorax. Their function is the prolonged monitoring of cardiac arrhythmias. All currently available event monitors are MRI compatible. It is recommended, at the physician's discretion, the evaluation of the data before the examination, due to the risk of loss of the information collected so far or even their suppression by the acquisition of magnetic field artifacts.

- Conditional devices: CIEDs for which exposure to magnetic field presents no risk to the patient. They are DCEIs that contain only electrode leads described by the manufacturer as conditional, connected to generators also described by the manufacturer as conditional and that do not meet the exclusion criteria. Electrode leads and generators must be from the same manufacturer. The use of electrode leads and generators from different manufacturers may not be guaranteed in case of damage to the system.

- Nonconditional devices: those that have not been extensively tested and/or are not guaranteed by the manufacturer against potential damage caused by the MRI environment (Table 2). 
Table 1. Pacemaker programming modes for performing nuclear magnetic resonance.

\begin{tabular}{|c|c|c|c|}
\hline Simulated chamber & Sensed chamber & Response to the sensed event & Adaptive frequency \\
\hline $\mathrm{O}=$ None & $\mathrm{O}=$ None & $\mathrm{O}=$ None & \\
\hline$A=$ Atrium & $A=$ Atrium & I = Inhibited & $\mathrm{R}=$ Connected frequency \\
\hline$V=$ Ventricle & $\vee=$ Ventricle & $\mathrm{T}=$ Triggered & responde \\
\hline $\mathrm{D}=$ Atrium and ventricle & $\mathrm{D}=$ Atrium and ventricle & $\mathrm{D}=$ Both & \\
\hline
\end{tabular}

Table 2. Checklist for performing magnetic resonance imaging (MRI) in patients with cardiac electronic devices.

\section{Before the examination}

Authorization from the surgeon informing (verification by the staff responsible for the MRI is necessary):

- If the device is conditional;

- If the autodetect or similar functions are programmed;

- If the programming will be performed by a member of the electrophysiologist team/member of the radiology service immediately before the MRI and after the end of the examination;

- If the patient is able to perform the procedure without reprogramming.

- If it complies with the institutional protocols:

- Check if there is a professional locally available for reprogramming the device when necessary (nonconditional or nonprogrammed);

- Check if there is staff capable of attending cardiorespiratory arrest (CRA);

- $\quad$ Check if there is material available for CRA care.

\section{During the examination}

- Check if there is effective rhythm and saturation monitoring.

- $\quad$ After the examination:

- $\quad$ Check the patient's vital signs;

- $\quad$ Check if the device has been reprogrammed with the approval of the physician responsible for the procedure;

- Confirm with the responsible physician the safety of the patient's discharge.

\section{MINIMUM SAFETY PARAMETERS}

The minimum safety parameters for patients with CIED in the MRI room are:

1. Monitoring of cardiac rhythm (preferably by electrocardiogram) and real-time saturation during the entire examination;

2. Presence of a physician and staff capable of attending cardiorespiratory arrest (CRA) in the radiology section (immediately outside zone 4, also known as the MRI room);

3. Availability of CRA material immediately outside zone 4, according to the current guidelines of the Advanced Cardiovascular Life Support (ACLS);

4. It is suggested to perform these procedures in a hospital or clinic that has parameters 1,2 and 3 and the ability to safely remove the patient to the intensive care unit in case of need;

5. Have an institutional standard operating protocol that is easily accessible to all laboratory members.

\section{SPECIFIC GUIDELINES FOR THE DIFFERENT DEVICES Conditional Stimulators}

Conditional implantable devices arrived in Brazil in 2012. The manufacturers initially developed electrode leads and generators that allow the performance of magnetic resonance exams with exclusion zone (avoiding thorax, cervical region and upper abdomen), subsequently developing authorized devices for the whole body. Other models were developed with similar technologies. 
The patient pacemaker identification card contains the model of his electrode leads and generator with information about compatibility with resonance imaging. The complete updated list of electrode leads and conditional generators is available on the website of the Brazilian Association of Arrhythmia, Electrophysiology and Artificial Heart Stimulation (ABEC) (https://abecdeca.org.br/medico).

However, CIEDs must be reprogrammed prior to exposure to the magnetic field. The objective of this reprogramming is to make the CIED indifferent to the magnetic field (asynchronous mode) and to perform other modifications, such as increasing the stimulation energy. Reprogramming should also evaluate command thresholds and remaining battery charge to assess the safety of the patient's exposure to the magnetic field. Ideally, the battery should not be less than $30 \%$ of its capacity and the control thresholds should not be raised prior to the test, although they are not absolute contraindications to the procedure.

Parameters of $1.5 \mathrm{~T}$, gradient slew rate $<$ or $=$ at $200 \mathrm{~T} / \mathrm{m} / \mathrm{s}$ and maximum SAR $<$ or $=$ at $2 \mathrm{~W} / \mathrm{kg}$ allow safety in all conditional devices regardless of region of interest. Some devices already allow $3 \mathrm{~T}$ and this can be verified if it in the interest of the patient and the physician responsible for imaging.

\section{Conditional Cardioverter-Defibrillators}

Conditional cardioverter-defibrillators also demonstrate proven safety to the patient's exposure to magnetic environment. They also need to be reprogrammed before the examination and return to the original parameters after its completion. In addition to indifference to the magnetic field (asynchronous mode), reprogramming aims to inhibit its inappropriate detection and its interpretation as tachycardia or ventricular arrhythmia.

This removes the antitachycardia protection inherent in the device during specific programming for resonance. Inhibition of tachycardia detection prevents the patient from receiving shocks during the examination.

Thus, in this context, if the patient spontaneously presents sustained ventricular tachycardia, treatment should be identical to that of patients who do not have an ICD, according to the current ACLS guidelines.

\section{Nonconditional Devices}

There is extensive literature on case series of patients with nonconditional CIED submitted to MRI without adverse events. Several studies are being conducted to validate the routine use of MRI in these devices.

Whenever MRI is the essential diagnostic method not replaceable or necessary on an emergency basis, the examination shall not be prevented by the presence of the CIED. Ideally, it should be carried out in an environment that meets the minimum conditions suggested in this document. Most devices submitted to MRI with $1.5 \mathrm{~T}$ tolerated the procedure, as well as exams lasting less than 40 minutes. Despite the absence of randomized studies, it is suggested the maintenance of safety parameters with short-term examinations and a field equal to or less than $1.5 \mathrm{~T}$.

Reprogramming of nonconditional CIED functionality to "resonance compatible" parameters may be performed on any CIED, even if the manufacturer does not ensure the safety of the components in case of damage, such as: reduction or suppression of cardiac stimulation, sudden mode change, heating of circuits and electrode leads, and failure to capture during or after the procedure.

If necessary to perform MRI in nonconditional CIED, it is suggested the presence of a physician able to evaluate the functioning and reprogramming of the device in the radiology environment, with reassessment of the CIED functionality at the end of the procedure and before discharge from the supervised environment. The need for temporary cardiac stimulation in case of device dysfunction should also be foreseen.

\section{EXCLUSION CRITERIA}

Exclusion criteria should be considered and discussed with the attending physician. In case of patients with absolute need for the examination in the presence of exclusion criteria, the risk of potentially fatal events should be discussed with 
the attending physician and the patient. The CIED shall be considered nonconditional and treated as such when there is (see description on nonconditional devices):

- Presence of abandoned or nonconditional electrode leads;

- Presence of epicardial electrode leads;

- Implant less than six weeks ago;

- Nonthoracic implants;

- Children.

\section{SUGGESTED ADDITIONAL LITERATURE}

IndikJH, Gimbel JR, Abe H, et al. HRS expert consensus statement on magnetic resonance imaging and radiation exposure in patients with cardiovascular implantable electronic devices Heart Rhythm. 2017;14(7):e97-e153. https://doi.org/10.1016/j.hrthm.2017.04.025

Mayo Foundation for Medical Education and Research. New protocols allow for MRI in selected patients with pacemakers [Internet]. Mayo Clinic; Disponível em: https://www.mayoclinic.org/medical-professionals/clinical-updates/cardiovascular/new-protocolsallow-mri-selected-pacemaker-patients

Mattei E, Gentili G, Censi F, et al. Impact of capped and uncapped abandoned leads on the heating of an MR-conditional pacemaker implant. Magn Reson Med. 2015;73(1):390-400. https://doi.org/10.1002/mrm.25106

Boilson BA, Wokhlu A, Acker NG, et al. Safety of magnetic resonance imaging in patients with permanent pacemakers: a collaborative clinical approach. J Interv Card Electrophysiol. 2012;33(1):59-67. https://doi.org/10.1007/s10840-011-9615-8

Burke PT, Ghanbari H, Alexander PB, et al. A protocol for patients with cardiovascular implantable devices undergoing magnetic resonance imaging (MRI): should defibrillation threshold testing be performed post-(MRI). J Interv Card Electrophysiol. 2010;28(1):5966. https://doi.org/10.1007/s10840-009-9463-y 